International Journal of Modern Physics A, Vol. 14, No. 7 (1999) 1151

(C) World Scientific Publishing Company

\title{
ERRATA
}

\section{LOW-ENERGY QUANTUM STRING COSMOLOGY}

\author{
[INT. J. MOD. PHYS. A, Vol. 13, No. 28 (1998) 4779-4786] \\ M. GASPERINI*
}

In the paper, Eq. (2.2) is incorrect and it should be

$$
g_{\mu \nu}=\operatorname{diag}\left(N^{2}(t),-a^{2}(t) \delta_{i j}\right), \quad a=\exp \left[\frac{\beta(t)}{\sqrt{d}}\right], \quad \phi=\phi(t),
$$

Eq. (3.1) is incorrect and it should be

$$
\left[\partial_{\bar{\phi}}^{2}-\partial_{\beta}^{2}+\lambda_{s}^{2} V(\beta, \bar{\phi}) e^{-2 \bar{\phi}}\right] \psi(\beta, \bar{\phi})=0 .
$$

*Permanent address: Dipartimento di Fisica, Università di Bari, Via Amendola 173, 70126 Bari, Italy. 